\title{
Excellent outcome after surgical treatment of massive pulmonary embolism in critically ill patients
}

\author{
Alexander Kadner, MD, Jürg Schmidli, MD, Florian Schönhoff, MD, Eva Krähenbühl, MD, Franz Immer, MD, \\ Thierry Carrel, MD, and Friedrich Eckstein, MD
}

From the Department for Cardiovascular Surgery, University Hospital Berne, Switzerland.

Received for publication Aug 9, 2007; revisions received Oct 30, 2007; accepted for publication Nov 13, 2007.

Address for reprints: Alexander Kadner, MD Clinic for Cardiovascular Surgery, University Hospital Berne, Freiburgstrasse, 3010 Berne, Switzerland (E-mail: alexander. kadner@web.de).

J Thorac Cardiovasc Surg 2008;136:448-51 0022-5223/\$34.00

Copyright $\odot 2008$ by The American Association for Thoracic Surgery

doi:10.1016/j.jtcvs.2007.11.021
Objective: Treatment of central and paracentral pulmonary embolism in patients with hemodynamic compromise remains a subject of debate, and no consensus exists regarding the best method: thrombolytic agents, catheter-based thrombus aspiration or fragmentation, or surgical embolectomy. We reviewed our experience with emergency surgical pulmonary embolectomy.

Methods: Between January of 2000 and March of 2007, 25 patients (17 male, mean age 60 years) underwent emergency open embolectomy for central and paracentral pulmonary embolism. Eighteen patients presented in cardiogenic shock, 8 of whom had cardiac arrest and required cardiopulmonary resuscitation. All patients underwent operation with mild hypothermic cardiopulmonary bypass. Concomitant procedures were performed in 8 patients ( 3 coronary artery bypass grafts, 2 patent foramen ovale closures, 4 ligations of the left atrial appendage, 3 removals of a right atrial thrombus). Follow-up is $96 \%$ complete with a median of 2 years (range, 2 months to 6 years).

Results: All patients survived the procedure, but 2 patients died in the hospital on postoperative days 1 (intracerebral bleeding) and 11 (multiorgan failure), accounting for a 30-day mortality of $8 \%$ (95\% confidence interval: 0.98-0.26). Four patients died later because of their underlying disease. Pre- and postoperative echocardiographic pressure measurements demonstrated the reduction of the pulmonary hypertension to half of the systemic pressure values or less.

Conclusion: Surgical pulmonary embolectomy is an excellent option for patients with major pulmonary embolism and can be performed with minimal mortality and morbidity. Even patients who present with cardiac arrest and require preoperative cardiopulmonary resuscitation show satisfying results. Immediate surgical desobstruction favorably influences the pulmonary pressure and the recovery of right ventricular function, and remains the treatment of choice for patients with massive central and paracentral embolism with hemodynamic and respiratory compromise.

$\mathrm{M}$ ortality rates after surgical pulmonary embolectomy in patients with acute central and paracentral pulmonary embolism have decreased in recent years. However, reported rates range from $6 \%$ to $40 \%$, and still reach up to $60 \%$ when the procedure is performed on critically ill patients. ${ }^{1-3}$ These high rates of unfavorable outcome have led to more conservative treatment recommendations for the majority of patients, reserving surgical embolectomy as a last-resort treatment for patients in severe shock and for patients in whom less-invasive strategies have failed. ${ }^{4}$

In contrast, a more aggressive surgical approach to massive pulmonary embolism has been reported by others, who find surgical embolectomy reasonable treatment for massive central and paracentral pulmonary embolism in case of hemodynamic compromise, but also in hemodynamically stable patients with right ventricular dysfunction. ${ }^{5,6}$

We present our institutional experience with the surgical treatment of central and paracentral pulmonary embolism. 


\section{Abbreviations and Acronyms \\ $\mathrm{CI}=$ confidence interval \\ $\mathrm{CPB}=$ cardiopulmonary bypass \\ $\mathrm{CT}=$ computed tomography}

\section{Materials and Methods}

Between January of 2000 and March of 2007, 25 patients underwent emergency surgical pulmonary embolectomy for acute massive pulmonary embolism. Seventeen patients were male with a mean age of 60 years (range 22-75 years). Patients' risk factors for the development of pulmonary embolism are shown in Table 1.

The most frequent indications to proceed with surgical embolectomy are presented in Table 2. All patients required inotropic support, and 18 patients $(72 \%)$ presented in cardiogenic shock with a shock index of greater than 1,8 (32\%) of whom experienced cardiac arrest and required cardiopulmonary resuscitation.

The diagnosis was made by contrast spiral computed tomography (CT) in all cases, showing bilateral and central clot burden (Figure 1). The localization of thrombi is summarized in Table 3. In addition, transthoracic echocardiography was performed in 19 patients and showed right ventricular dysfunction and pulmonary hypertension (defined as a right ventricular/right atrial gradient $>30 \mathrm{~mm} \mathrm{Hg}$ ) in all of these patients.

\section{Surgical Technique}

Surgical embolectomy is performed through a median sternotomy on mild hypothermic cardiopulmonary bypass (CPB) using bicaval cannulation. The main pulmonary artery is opened with a longitudinal incision, which is extended into the right or left pulmonary artery branches if necessary. The thrombotic material is extracted by means of forceps and assisting suction. To allow for complete clot removal, the left and right pulmonary artery branches were videoscopically inspected up to the segmental arteries in 8 cases. Massage of the lungs is not performed to avoid additional damage to the lung parenchyma. Furthermore, the right atrium and ventricle are explored routinely, all clot material is carefully removed, and a patent foramen ovale is closed in every patient. The pulmonary arteriotomy is closed with a running suture, and the patients are weaned from CPB after declamping.

\section{Results}

The median CPB time was 74 minutes (range, 47-144 minutes), and the median aortic crossclamp time was 41 minutes (range, 27-95 minutes). Three patients underwent beatingheart surgery. Concomitant procedures were performed in 8 patients ( 3 coronary artery bypass grafts, 2 patent foramen ovale closures, 4 ligations of the left atrial appendage, 4 removals of a right atrial thrombus).

All patients survived the procedure. The 30-day mortality was $8 \%$ ( 2 patients) (95\% confidence interval [CI]: 0.9826.03). A 62-year-old female patient died of intracerebral bleeding on postoperative day 1 . She was referred after 2 unsuccessful attempts of thrombolysis. A 70-year-old male patient died of aspiration pneumonia and multiorgan failure on
TABLE 1. Risk factors for development of pulmonary embolism $(n=25)$

\begin{tabular}{ll}
\hline \multicolumn{1}{c}{ Condition } & $\mathbf{n ( \% )}$ \\
\hline Deep vein thrombosis & $8(32 \%)$ \\
Immobility & $7(28 \%)$ \\
Cancer & $4(16 \%)$ \\
Pregnancy & $1(4 \%)$ \\
Smoking history & $4(16 \%)$ \\
Hypercoagulability & $1(4 \%)$ \\
none & $2(8 \%)$ \\
\hline
\end{tabular}

postoperative day 11 . This patient had also undergone unsuccessful thrombolytic therapy before surgical intervention. Both patients had undergone cardiopulmonary resuscitation and presented preoperatively in profound cardiogenic shock with maximal inotropic support and shock indexes of 3.3 and 2.1 , respectively.

Reoperations for mediastinal bleeding were performed in 2 patients (8\%). Postoperatively, all patients received anticoagulation therapy starting with heparin 6 hours postoperatively until oral anticoagulation with warfarin was fully effective with a target international normalized ratio between 2.0 and 3.0. No filters were inserted into the inferior vena cava.

The median follow-up interval was 24 months (range, 2-85 months) and is complete in 96\% (24 patients). Twelve patients were followed for more than 2 years, 9 patients were followed for more than 3 years, and 7 patients were followed for more than 4 years.

There were 4 late deaths. Actuarial survival at 1 and 4 years' follow-up was $80 \%$ (95 CI: 0.61-0.93) at 1 year and $76 \%$ (95 CI: $0.54-0.9)$ at 4 years. All patients with late deaths (at 2, 4, and 5 months, and at 3 years) died of cancer.

Postoperative echocardiographic pressure measurements demonstrated a significant reduction $(P<.001)$ of the pulmonary pressure to half of the systemic values or less in all patients $(n=13)$ who received preoperative and postoperative echocardiographic evaluation with estimation of the pulmonary artery pressure (Figure 2). At last follow-up, all patients were classified with New York Heart Association

TABLE 2. Indications for surgical embolectomy $(n=25)$

\begin{tabular}{lc}
\hline \multicolumn{1}{c}{ Indication } & $\mathbf{n ~ ( \% )}$ \\
\hline Active bleeding & $1(4 \%)$ \\
Recent surgical interventions & $4(16 \%)$ \\
Stroke & $1(4 \%)$ \\
Failure of thrombolytics & $4(16 \%)$ \\
Failure of catheter embolectomy & $1(4 \%)$ \\
Large RA-RV thrombus & $4(16 \%)$ \\
RV hemodynamic dysfunction & $19(76 \%)$ \\
Cardiogenic shock & $18(72 \%)$ \\
Cardiac arrest & $8(32 \%)$ \\
\hline
\end{tabular}

$R A$, Right atrial; $R V$, right ventricular. 


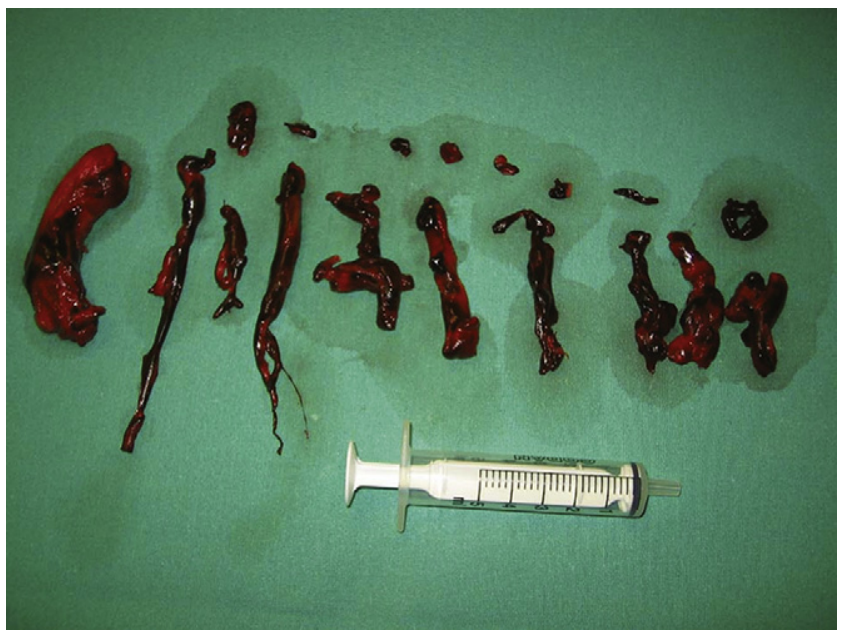

Figure 1. Embolic clot removed from a patient with bilateral massive pulmonary embolism.

functional class I or II, and no recurrence of pulmonary embolism was observed.

\section{Discussion}

Early mortality after surgical pulmonary embolectomy is reported to be high. Published reports state that the rate has improved, ranging from $8 \%$ to $27 \% .^{5-7}$ However, mortality rates are found to increase significantly to $30 \%$ to $40 \%$ when surgical pulmonary embolectomy is performed in patients who present in critical preoperative conditions. Consequently, patient selection and the level of aggressiveness pursuing with open pulmonary embolectomy remain unclear and a subject of debate. Criteria recommended for pulmonary embolectomy are 1) massive pulmonary embolism, angiographically documented if possible; 2) hemodynamic instability (shock) despite heparin therapy and resuscitation efforts; and 3) the failure of thrombolytic therapy or a contraindication to its use (4). We think that the criteria of failure of thrombolytic therapy is critically affecting these patients by delaying surgery, and a substantial number of them require massive inotropic support or even cardiopulmonary resuscitation before surgery.

TABLE 3. Localization of thrombi $(n=25)$

\begin{tabular}{lc}
\hline \multicolumn{1}{c}{ Localization } & n (\%) \\
\hline Right atrium & $4(16 \%)$ \\
Patent foramen ovale & $2(8 \%)$ \\
Left atrium & $1(4 \%)$ \\
Main pulmonary artery and bilateral extension & $18(72 \%)$ \\
Paracentrally in left and right pulmonary artery & $7(28 \%)$ \\
Superior vena cava & $1(4 \%)$ \\
Inferior vena cava & $1(4 \%)$ \\
\hline
\end{tabular}

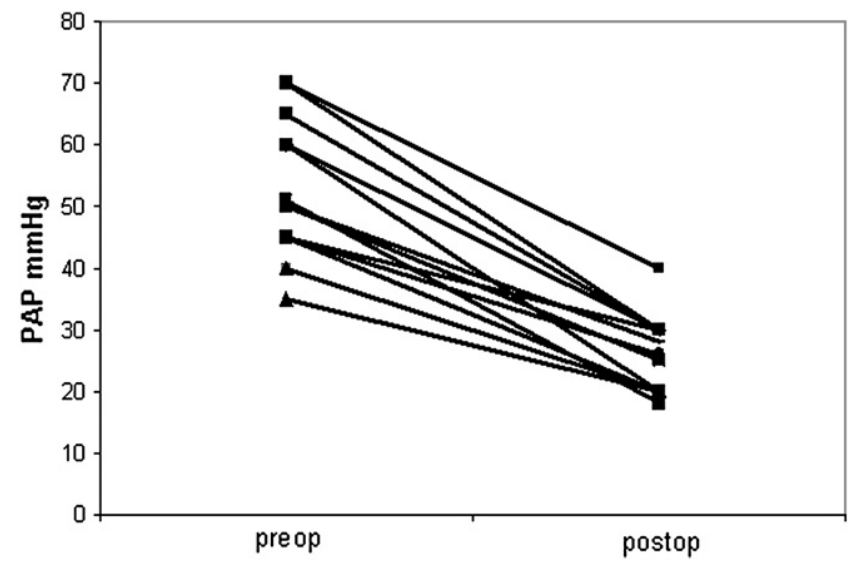

Figure 2. Pre- and postoperative measurement of pulmonary artery pressure. Pre- and postoperative echocardiographic measurements with estimation of the pulmonary artery pressure were performed in 13 patients and demonstrated the reduction of the pulmonary hypertension to half systemic values or less in all of these patients. PAP, Pulmonary artery pressure.

Others suggest a more aggressive approach. Leacche and colleagues ${ }^{6}$ recommend surgical embolectomy in the presence of large central clot burden in hemodynamically stable patients who show right ventricular dysfunction. They report on 47 patients with an operative mortality rate of $6 \%$ (3 patients). Two of their patients had preoperative cardiac arrest, and there were 6 late deaths $(12 \%)$, mostly from metastatic cancer.

Our observation compares favorably with these data, with all patients surviving the procedure and a 30-day mortality of $8 \%$ (2 patients). Both early postoperative deaths were not directly related to surgery; 1 patient died of intracerebral bleeding on postoperative day 1 , and 1 patient died of multiorgan failure on postoperative day 11. Both patients had undergone two unsuccessful attempts of thrombolytic therapy.

Both patients who died early in this study had preoperative cardiac arrest, and this underlines the strong negative impact of preoperative cardiac arrest on the operative mortality rate after pulmonary embolectomy. In a recent systematic review of more than 40 reports, Stein and colleagues ${ }^{8}$ found a $59 \%$ operative mortality rate among patients with cardiac arrest before pulmonary embolectomy. In this study, a 29\% mortality rate ( 2 deaths) was found among the 7 patients experiencing preoperative cardiac arrest.

With regard to late death, we observed a $16 \%$ late mortality rate caused by the 4 patients' underlying diseases, primarily metastatic cancer.

The cause of early death in patients undergoing pulmonary embolectomy has been largely attributed to right ventricular failure secondary to persistent pulmonary hypertension, interstitial pulmonary edema, and massive parenchymal and intrabronchial hemorrhage. ${ }^{9,10}$ A proposed explanation is 
an incomplete removal of thrombotic material from the distal pulmonary arterial tree causing persistent hypertension.

The extraction of distal thrombotic material is commonly attempted by means of Fogarty catheter extraction or manual compression of the lungs. Both methods cannot be well controlled and may cause mechanical injuries to the pulmonary arterial wall, especially in the segmental branches, and may provoke parenchymal and endobronchial bleeding. Spagnolo and colleagues ${ }^{11}$ proposed retrograde pulmonary perfusion to flush out residual thrombotic material and to prevent pulmonary air embolism by a retrograde filling of the pulmonary artery. We do not find this technique necessary and did not use it in any of our patients. We found pulmonary arterioscopy to be a useful technique to localize and facilitate distal clot extraction. We think that the most complete removal of thrombotic material is the best predictor to avoid persistent pulmonary artery hypertension.

In the situation of more peripherally located clot burden and difficult removal, cooling on CPB of the patient could also be considered to reduce CPB flow for an improved visualization. However, this additional maneuver was not necessary in our patients.

In regard to the postoperative anticoagulation therapy of the patients, heparin was started 6 hours after surgery and continued until warfarin was fully effective with a target international normalized ratio between 2.0 and 3.0. No caval filters were placed postoperatively. Caval filters might prevent recurrent embolism, but patients with filters are prone to develop deep vein thrombosis. ${ }^{12,13}$ Therefore, the placement of temporary caval filters might be considered in patients who have transient contraindications to anticoagulation therapy and who experience recurrent embolism despite optimal oral anticoagulation.

In contrast with many previous studies, our results confirm that surgical pulmonary embolectomy can be performed with a low mortality rate in patients with massive central and paracentral pulmonary embolism, even in those who experience cardiac arrest before surgical intervention. We think that an essential component for this improved outcome is a multidisciplinary approach with rapid diagnosis by $\mathrm{CT}$ and echocardiography. CT helps to identify patients with centrally localized emboli, which are surgically accessible, whereas patients presenting with the majority of the clot burden located more peripherally might not be good candidates for a surgical intervention. Echocardiography allows the identification of patients with impending right ventricular dysfunction, which has been shown to be a strong risk factor for death.

In view of the low mortality rate revealed by more recent reports, including the present one, and the lower number of hemorrhagic events and lower rate of recurrent pulmonary embolic events when compared with medical treatment in hemodynamically compromised patients, surgical embolectomy should not be reserved for patients with massive pulmonary embolism who present in cardiogenic shock, as advocated in the past. Unfortunately, the Trendelenburg procedure (using inflow occlusion without $\mathrm{CPB}$ ) might still be considered by emergency department doctors. However, this is a heroic surgical intervention that has nothing in common with the meticulous extraction of the thrombotic material, as currently practiced.

\section{Conclusions}

This report shows that good results can be achieved with surgery in patients with pulmonary embolism and hemodynamic instability. A multidisciplinary approach is an essential component of the management, and the application of angioscopy allows for an optimized clot removal. Furthermore, extension of the indication for surgical pulmonary embolectomy should be considered beyond the traditional indications, such as failure of lytic treatment or hemodynamic compromise, even to hemodynamically stable patients with right ventricular dysfunction and a high clot burden in the central pulmonary arteries.

\section{References}

1. Gray HH, Miller GA, Paneth M. Pulmonary embolectomy: its place in the management of pulmonary embolism. Lancet. 1988;1:1441-5.

2. Meyer G, Tamisier D, Sors H, Stern M, Vouhe P, Makowski S, et al. Pulmonary embolectomy: a 20-year experience at one center. Ann Thorac Surg. 1991;51:232-6.

3. Kucher N, Goldhaber SZ. Risk stratification of acute pulmonary embolism. Semin Thromb Hemost. 2006;32:838-47.

4. Buller HR, Agnelli G, Hull RD, Hyers TM, Prins MH, Raskob GE. Antithrombotic therapy for venous thromboembolic disease: the Seventh ACCP Conference on Antithrombotic and Thrombolytic Therapy. Chest. 2004;126:401S-28S.

5. Aklog L, Williams CS, Byrne JG, Goldhaber SZ. Acute pulmonary embolectomy: a contemporary approach. Circulation. 2002;105:1416-9.

6. Leacche M, Unic D, Goldhaber SZ, Rawn JD, Aranki SF, Couper GS, et al. Modern surgical treatment of massive pulmonary embolism: results in 47 consecutive patients after rapid diagnosis and aggressive surgical approach. J Thorac Cardiovasc Surg. 2005;129:1018-23.

7. Digonnet A, Moya-Plana A, Aubert S, Flecher E, Bonnet N, Leprince P, et al. Acute pulmonary embolism: a current surgical approach. Interact Cardiovasc Thorac Surg. 2007;2:27-9.

8. Stein PD, Alnas M, Beemath A, Patel NR. Outcome of pulmonary embolectomy. Am J Cardiol. 2007;99:421-3.

9. Jakob H, Vahl C, Lange R, Micek M, Tanzeem A, Hagl S. Modified surgical concept for fulminant pulmonary embolism. Eur J Cardiothorac Surg. 1995;9:557-61.

10. Shimokawa S, Uehara K, Toyohira H, Saigenji H, Moriyama Y, Taira A, et al. Massive endobronchial hemorrhage after pulmonary embolectomy. Ann Thorac Surg. 1996;61:1241-2.

11. Spagnolo S, Grasso MA, Tesler UF. Retrograde pulmonary perfusion improves results in pulmonary embolectomy for massive pulmonary embolism. Tex Heart Inst J. 2006;33:473-6.

12. White RH, Zhou H, Kim J, Romano PS. A population-based study of the effectiveness of interior vena cava filter use among patients with venous thromboembolism. Arch Intern Med. 2000;160:2033-41.

13. Decousus H, Leizorovicz A, Parent F, Page Y, Tardy B, Girard P, et al. A clinical trial of vena caval filters in the prevention of pulmonary embolism in patients with proximal deep-vein thrombosis. Prevention du Risque d'Embolie Pulmonaire par Interruption Cave Study Group. N Engl J Med. 1998;338:409-15. 